\title{
Burden of hospitalization for heart failure in the United States: a systematic literature review
}

\author{
Katherine M Osenenko, MCPM; Effie Kuti, PharmD; Alison M Deighton, BSc; Pratik Pimple, PhD;
} and Shelagh M Szabo, MSc

\section{What is already known about this subject}

- Heart failure (HF) is highly prevalent and is associated with considerable morbidity and mortality; the prevalence of $\mathrm{HF}$ is projected to increase.

- The economic burden of HF is substantial and hospitalizations for $\mathrm{HF}$ (HHF) are the largest component of associated direct medical costs.

- Although several recent reviews have focused on the broader economic burden associated with $\mathrm{HF}$, and 1 recent review included a synthesis of cost estimates for HHF, the relationship between costs and clinical outcomes associated with $\mathrm{HHF}$ is unclear.

\section{ABSTRACT}

BACKGROUND: Heart failure (HF) affects approximately 6 million Americans, with prevalence projected to increase by $46 \%$ and direct medical costs to reach $\$ 53$ billion by 2030. Hospitalizations are the largest component of direct costs for HF; however, recent syntheses of the economic and clinical burden of hospitalization for heart failure (HHF) are lacking.

OBJECTIVE: To synthesize contemporary estimates of cost and clinical outcomes of $\mathrm{HHF}$ in the United States.

METHODS: A systematic literature review was conducted using MEDLINE and Embase to identify articles reporting cost or charge

\section{What this study adds}

- This systematic literature review was conducted to synthesize contemporary estimates of cost per HHF in the United States and associated clinical outcomes, including length of stay, risk of rehospitalization, and case fatality.

- This synthesis demonstrates the substantial economic burden of $\mathrm{HHF}$, with mean HF-specific inpatient costs ranging from $\$ 10,737$ to $\$ 17,830$ per hospitalization.

- Factors contributing to variability in estimates include length of stay, age and sex of the sample, HF severity, and frequencies of comorbidities.

\section{Author affiliations \\ Katherine M Osenenko, MCPM; Alison M Deighton, BSc; and Shelagh M Szabo, MSc, Broadstreet HEOR, Vancouver BC Canada. Effie Kuti, PharmD, and Pratik Pimple, PhD, Boehringer Ingelheim Pharmaceuticals, Ridgefield, CT.}

AUTHOR CORRESPONDENCE: Shelagh M Szabo, 604.800.0648; sszabo@broadstreetheor.com per HHF in the United States published between January 2014 and May 2019. Subgroups of interest were those with both $\mathrm{HF}$ and renal disease or diabetes, as well as $\mathrm{HF}$ with reduced or preserved ejection fraction (HFrEF or HFpEF).

RESULTS: 23 studies reporting cost and/or charge per HHF were included. Sample sizes ranged from 989 to approximately 11 million (weighted), mean age from 65 to 83 years, and $39 \%$ to $74 \%$ were male. Cost per HHF ranged from $\$ 7,094$ to $\$ 9,769$ (median) and $\$ 10,737$ to $\$ 17,830$ (mean). Charge per HHF ranged from $\$ 22,162$ to $\$ 40,121$ (median), and $\$ 50,569$ to $\$ 50,952$ (mean). Among patients with renal disease, HHF mean cost ranged from $\$ 9,922$ to $\$ 41,538$. For those with HFrEF or HFpEF, mean cost ranged from
$\$ 11,600$ to $\$ 17,779$ and $\$ 7,860$ to $\$ 10,551$, respectively. No eligible studies were identified that reported HHF costs or charges among patients with $\mathrm{HF}$ and diabetes. Cost and charge per HHF increased with length of stay, which ranged from 3 to 5 days (median) and 4 to 7 days (mean).

CONCLUSIONS: This synthesis demonstrates the substantial economic burden of $\mathrm{HHF}$ and the variability in estimates of this burden. Factors contributing to variability in estimates include length of stay, age and sex of the sample, HF severity, and frequencies of comorbidities. Further research into cost drivers of $\mathrm{HHF}$ is warranted to understand potential mechanisms to reduce associated costs. 
Heart failure (HF) is highly prevalent and is associated with considerable morbidity and mortality. It is estimated that approximately 6 million individuals in the United States had HF between 2015 and 2018, ${ }^{1}$ and prevalence is projected to increase by 46\% between 2012 and 2030, affecting up to 1 in 33 individuals. ${ }^{2}$ Although HF incidence has decreased in recent years, the projected increase in prevalence and recent improvement in survival rates are due to advancements in HF treatment. ${ }^{3}$ Despite these improvements, the 1-year mortality rate remains high at approximately $30 \%$, increasing to approximately $40 \%$ at 5 years. ${ }^{4}$ A 2018 US analysis of data from the Healthcare Cost and Utilization Project Nationwide Emergency Department and Inpatient Samples and the Vital Statistics System reported that the number of unique acute clinical events (including emergency department [ED] visits, hospitalizations, and deaths) with HF as the primary diagnosis had decreased since 2006. However, events with HF as a secondary or contributing diagnosis increased over the same period, leading to an overall increase in the rate of HF-related events. ${ }^{5}$

The corresponding economic burden of HF is substantial, with total combined direct and indirect costs of HF estimated at \$30.7 billion US dollars (USD) in 2012 based on Medical Expenditure Panel Survey data, of which \$20.9 billion and $\$ 9.8$ billion are attributed to direct medical costs and indirect costs, respectively. ${ }^{2}$ In 2014, hospitalization costs alone were estimated at $\$ 11$ billion, with a mean cost per hospitalization of $\$ 11,552 .^{5}$ Along with increasing prevalence, the American Heart Association has forecasted that total direct medical costs of HF will increase from \$21 billion in 2012 to \$53 billion in 2030 (2010 USD). ${ }^{2}$ Hospitalizations are the largest component of direct medical costs for $\mathrm{HF}$, with estimates ranging from $49 \%$ to $73 \%$ of total costs. ${ }^{6}$ However, variations exist between estimates of HF-related costs, including for hospitalizations. A recent US analysis reported disparities in clinical and economic burden of HF across US states, ${ }^{7}$ highlighting the need to understand not only the economic burden of $\mathrm{HF}$, but also the drivers of this burden.

As hospitalizations contribute substantially to overall HF costs, improved understanding of costs associated with hospitalizations for HF (HHF), as well as clinical outcomes of those hospitalizations, is important to better understand the burden associated with HF overall. Although several recent reviews have focused on the broader economic burden associated with $\mathrm{HF}$, including a recent review synthesizing the cost estimates for $\mathrm{HHF}^{8}$ the relationship between costs and clinical outcomes associated with HHF remains unclear. There is a need for a synthesis of contemporary estimates of the economic burden of $\mathrm{HHF}$, with nuanced consideration of the drivers of HHF costs in the United States and resulting implications for projections and planning.

To fill this gap, the objective of this review was to synthesize contemporary estimates of cost per HHF in the United States. Secondary objectives were to synthesize estimates of (1) clinical outcomes, including length of stay (LOS), rehospitalization, and case fatality (percentage of patients who died during the HHF episode) or mortality, and (2) HHF cost among the subgroups of patients with both $\mathrm{HF}$ and renal disease (eg, chronic kidney disease [CKD]) or diabetes, as well as by reduced ejection fraction (HFrEF) or preserved ejection fraction (HFpEF) status.

\section{Methods}

A systematic literature review (SLR) was conducted using MEDLINE and Embase to identify articles that reported estimates of cost or charge per HHF in the United States. The search was limited to articles published within the past 5 years (January 2014-May 2019) and conference abstracts from the last 2 years (January 2017-May 2019). The search strategy included terms related to HF and its treatment, economic burden, and costs and hospitalization, as well as study design filters (Supplementary Table 1, available in online article). The SLR study design was guided by the PICOS (Population, Intervention, Comparators, Outcomes, Study Design) criteria (Supplementary Table 2, available in online article). Animal studies, along with non-US studies, were excluded, and articles were limited to those published in English.

Abstracts retrieved from the search strategy were independently screened by 2 reviewers, as were full-text articles identified for inclusion following abstract screening. Consensus was reached through discussion where reviewers initially disagreed on whether a record should be included. Reference lists of identified review articles were searched for records meeting the study's PICOS criteria.

Double data extraction was performed for demographic, economic, and clinical outcomes data of interest from the eligible studies. Any discrepancies observed between the 2 reviewers were resolved through discussion to achieve consensus. For continuous variables, the mean, median, SD, and range were extracted whenever available. For dichotomous and categorical variables, the number of individuals and proportion were extracted. Data extracted from each study included study author and year of publication; study design (including details of methodology, cost calculation methods, sampling frame, and case ascertainment, as applicable); population; baseline clinical and demographic characteristics (eg, payer type and key comorbidities at baseline); sample size; economic burden outcomes (eg, cost 


\section{FIGURE 1 PRISMA Diagram}

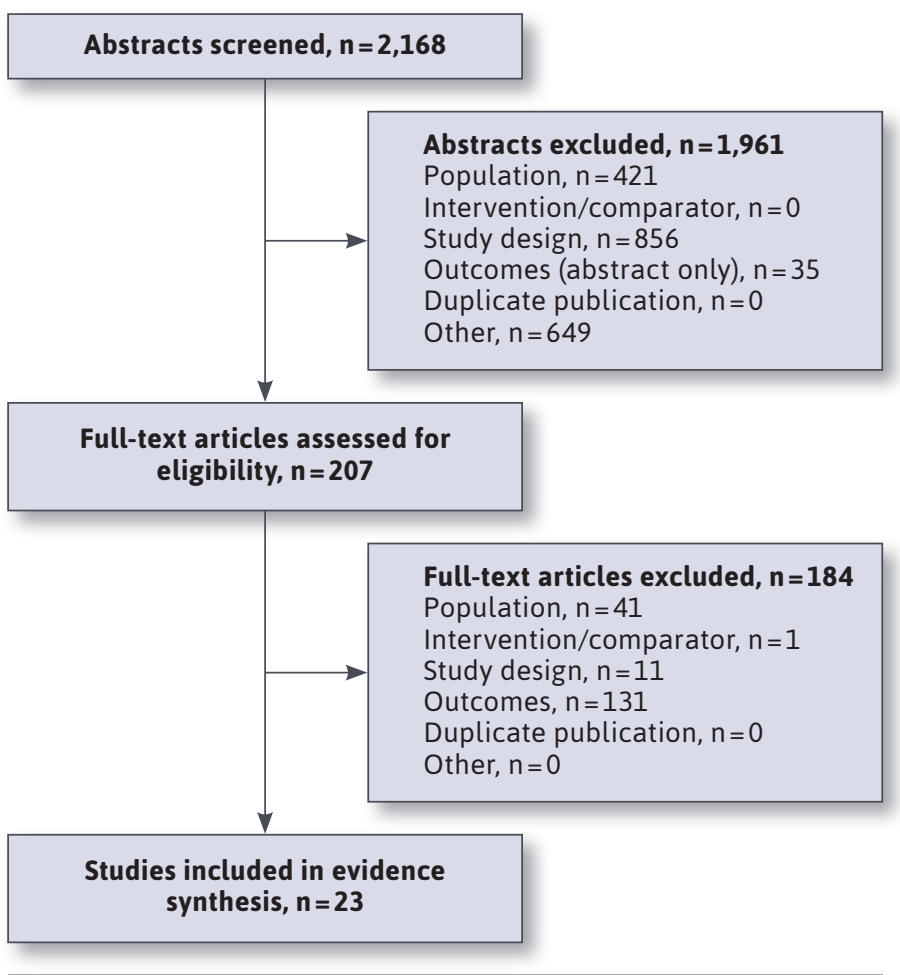

PRISMA = Preferred Reporting Items for Systematic Reviews and Meta-Analyses.

or charge per HHF); epidemiology estimates; and clinical outcomes (eg, LOS).

The primary focus was on the cost per HHF episode. Other outcomes of interest were extracted for those papers reporting the primary outcome of interest. Costs and charges for HHF were considered-costs represent the actual cost of providing the hospital services, and charges reflect the amount that is billed to the patient or insurer for those services. ${ }^{9}$ Charges are often reported as a proxy estimate in the absence of direct cost data; however, it should be noted that they are not reflective of the actual economic burden. ${ }^{9}$

Patient and study characteristics were summarized using counts and proportions, and means/medians with standard deviations (SDs), standard errors (SEs), 95\% confidence intervals (CIs), or interquartile ranges (IQRs), as appropriate. Patient characteristics included age at baseline, percentage of the sample that was male, percentage of the sample by payer type, and percentage that had key comorbidities associated with HF at baseline (eg, diabetes, kidney disease, cerebrovascular disease, obesity, or hypertension). Study characteristics included study inclusion criteria, period, design, database, and whether costs or charges were reported. Study quality assessment was performed using the STROBE (Strengthening the Reporting of Observational Studies) criteria (Supplementary Table 3, available in online article). ${ }^{10}$

Costs and charges per HHF were summarized overall and by component cost, where reported, using means or medians with SD, SE, or IQR, as reported. All costs and charges were converted to 2019 USD using US health care specific Consumer Price Index values. ${ }^{11}$ Costs and charges overall were plotted as histograms by study citation and as scatter plots by key patient characteristics (eg, age at baseline and the percentage of the sample that was male). An estimate of the total annual costs of HHF in the United States was calculated based on the annual number of hospitalizations for $\mathrm{HF}$ and the maximum mean cost per HHF identified in this review.

Among studies reporting costs and/or charges per HHF, data on selected clinical outcomes were summarized in tabular form. LOS estimates were summarized using means or medians with SD, SE, or IQR. Rehospitalization, stratified by all-cause or HF-specific rehospitalization, was summarized at 30-day, 60-day, 90-day, and 1-year intervals in tabular form. Case fatality was presented as the percentage of patients who died during the index HF hospitalization episode.

Details of economic and clinical outcomes were summarized among patient subgroups of interest (renal disease; HFrEF vs HFpEF), where available.

\section{Results}

\section{STUDY CHARACTERISTICS}

From 2,177 records identified, 9 duplicates were removed. From the 2,168 abstracts screened, 1,961 that did not meet the eligibility criteria were excluded (Figure 1). Of the remaining 207 records, 184 were ineligible and excluded on full-text review, leaving 23 eligible studies reporting cost ( $n=17$ studies) or charge $(n=4)$ per HHF included; 2 studies reported both cost and charge (Figure 1 and Supplementary Table 4 , available in online article). Publication dates ranged from 2014 to 2019. Twenty-one retrospective observational studies,,${ }^{5,12-31} 1$ cross-sectional database study, ${ }^{32}$ and 1 singlecenter population-based study ${ }^{33}$ were identified. Two of the 23 included studies were conference abstracts, ${ }^{23,24}$ and the remaining 21 were full-text manuscripts. Findings from the study quality assessment are shown in Supplementary Table 5 (available in online article). 


\section{FIGURE 2 Mean and Median Cost per HHF}

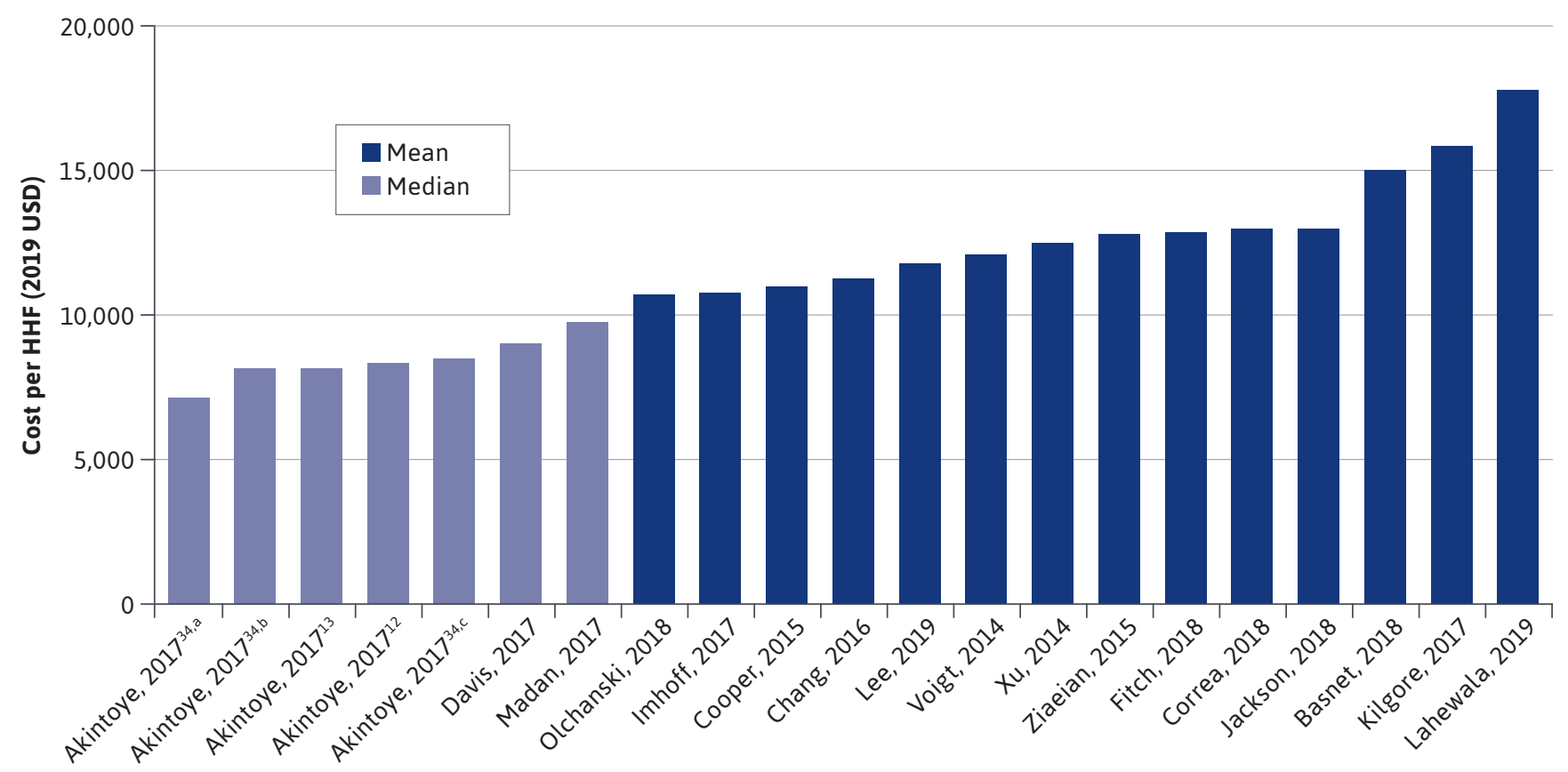

aPrivate hospitals (invest).

${ }^{b}$ Government hospitals.

'Private hospitals (nonprofit).

$\mathrm{HHF}=$ hospitalization for heart failure; USD= United States dollars.

Across included studies, sample sizes ranged from 989 to approximately 11 million (weighted) HF patients (Supplementary Table 4). ${ }^{17,20}$ The mean age of the samples ranged from 65.0 to 83.3 years, ${ }^{5,17}$ and the percentage of males ranged from $38.7 \%$ to $74.0 \% .^{17,25}$ Among studies reporting the distribution of primary insurance payer type, Medicare was the most frequently reported primary payer. The percentage of patients with Medicare as the primary payer ranged from $58 \%$ to $100 \%$ 17,19,22,25; the percentage with primary commercial coverage ranged from $7 \%$ to $17 \% \%^{18,27}$; the percentage with primary Medicaid coverage ranged from $8 \%$ to $12 \%$, 27,32 ; and the percentage with primary private coverage ranged from $3 \%$ to $23 \% .{ }^{17,18}$ Among the studies that reported on the percentage of individuals who had key HF-related comorbidities, the percentage of patients with diabetes ranged from $29.0 \%$ to $47.9 \%,{ }^{5,25}$ with kidney disease from $0.2 \%$ to $55.7 \%,{ }^{18,29}$ with cerebrovascular disease from $1.0 \%$ to $13.5 \%,{ }^{25,33}$ with obesity from $14.3 \%$ to $21.0 \%,{ }^{16,33}$ and with hypertension from $18.3 \%$ to $81.3 \% .^{24,29}$ Three studies reported results for the subgroup of individuals with both
HF and renal disease, ${ }^{20,24,30}$ and 4 studies reported results for HFrEF or HFpEF subgroups. ${ }^{17,27,30,33}$ No studies included in this review reported on the cost per hospitalization for the subgroup of patient with both HF and diabetes.

\section{ECONOMIC BURDEN OF HHF}

Mean (SD) cost per HHF ranged from $\$ 10,737$ (\$12,044) among patients with acute decompensated HF (mean age, 71.1 years; single-center population-based study) to $\$ 17,830(\$ 47,713)$ among a sample of adults who were rehospitalized for HF (mean age, not reported [NR]; Nationwide Readmissions Database; Figure 2). ${ }^{26,33}$ Median (IQR) cost per HHF ranged from $\$ 7,094$ ( $\$ 5,054$; mean age, 72.0 years; National Inpatient Sample [NIS]) to $\$ 9,769$ (\$19,171; among a sample of whom $65 \%$ were aged $\geq 70$ years; Statewide Planning and Research Cooperative System [SPARCS]; Figure 2). ${ }^{28,34}$ Two studies identified in the review reported on the mean (SE) charge per HHF: \$50,569 (\$895; mean age, 72.1 years) and \$50,952 (\$437; mean age, 71.8 years; Figure 3). . $^{53}$

These studies were conducted using NIS data in 20062014 and 2016; when inflated to 2019 USD, charges were 


\section{FIGURE 3 Mean and Median Charges per HHF}

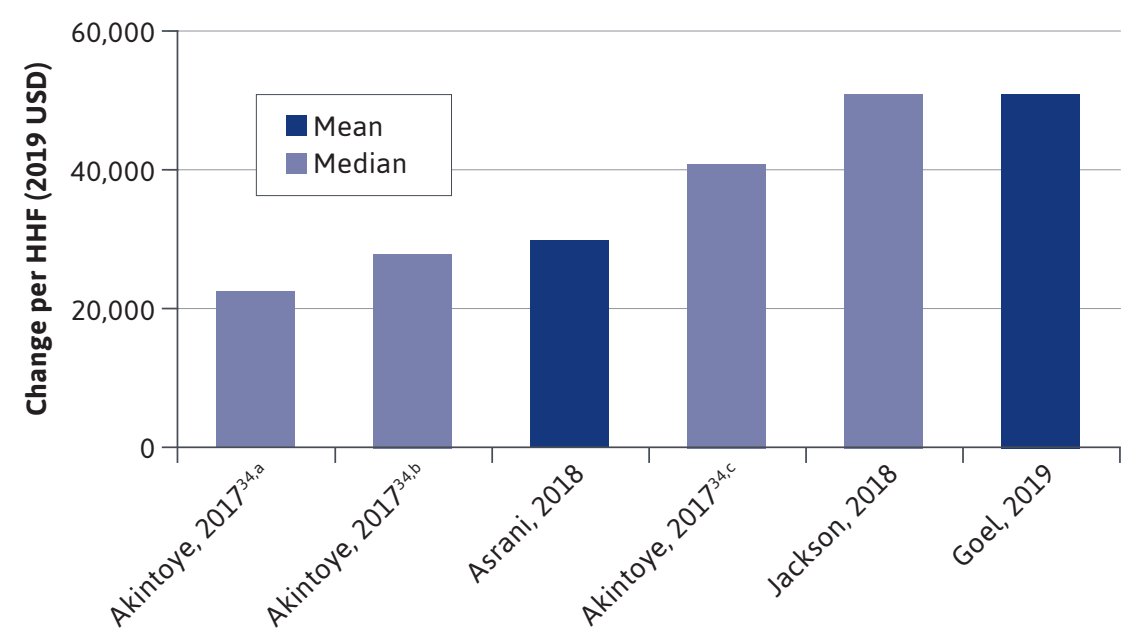

aGovernment hospitals.

bPrivate hospitals (nonprofit).

'Private hospitals (invest).

$H H F=$ hospitalization for heart failure; USD = United States dollars.

very similar between the 2 studies. Only 1 study, using NIS data, reported median (IQR) charge data, and estimates per HHF ranged from $\$ 22,162$ (\$27,293; among patients seen at government-owned hospitals) to $\$ 40,121$ (\$45,065; among patients seen at investor-owned hospitals; Figure 3). ${ }^{34}$ This study was the only one to report costs or charges by hospital ownership type. While 3 studies included exclusively Medicare populations, ${ }^{19,22,25}$ the remaining studies included populations with a mixture of primary payer types but did not report costs or charges separately by payer type. Cost and charge per HHF increased with LOS (Table 1).

The distribution of costs and charges data per HHF were explored according to mean baseline age (Supplementary Figure 1, available in online article), the percentage of the sample that was male (Supplementary Figure 2, available in online article), and the percentage with various comorbidities (data not shown); however, no clear relationships were identified. Based on the annual number of HHF in the United States $(n=978,135)$ and the maximum mean cost per HHF $(\$ 17,830)$ identified in this review, ${ }^{5,26}$ it is estimated that inpatient costs alone could translate up to $\$ 18$ billion annually in the United States.

Among patients with $\mathrm{HF}$ and renal disease, mean (SD) costs per HHF ranged from $\$ 9,922$ (NR; patients with CKD and history of stroke; mean age, 76.9 years; NIS) to $\$ 41,538$ (NR; patients with acute kidney injury requiring dialysis [D-AKI]; mean age, 69.3 years; NIS; Figure 4). ${ }^{20,24}$ Mean (SE) cost per HHF among patients with HFpEF ranged from $\$ 7,860$ (\$6,937; mean age, NR; University Health System Consortium [UHC] database) to $\$ 10,551(\mathrm{NR}$; mean age, NR; NIS). ${ }^{27,30}$ Among those with HFrEF, mean (SD) cost per HHF ranged from \$11,600 (\$13,533; mean age, 67.5 years; single-center population-based study) to $\$ 17,779$ (SE: $\$ 6,937$; mean age, NR; UHC database). ${ }^{27,33}$ One study reported a mean (SD) charge per HHF of $\$ 65,251$ $(\$ 124,373)$ among adults with HFrEF (mean age, 65.0; NIS). ${ }^{17}$

No studies reported cost or charge per HHF for patients with both HF and diabetes. The distribution of costs and charges data per HHF among patients with renal disease, HFpEF, or HFrEF were explored according to mean baseline age (Supplementary Figure 3, available in online article), the percentage of the sample that was male (Supplementary Figure 4, available in online article), and the percentage with various comorbidities (data not shown); however, no clear relationships were identified.

In addition to HHF costs and charges overall, the cost per ED visit was reported separately from other inpatient charges in 1 study (mean $[\mathrm{SE}], \$ 1,310[\mathrm{NR}]) .{ }^{22}$ The charge per ED visit was reported separately from other inpatient costs in 2 studies (mean [SE], \$3,526 [\$97]; median [IQR], \$1,905 [8,083]). ${ }^{29}$

Because studies in this systematic review were selected based on patient hospitalization for $\mathrm{HF}$, it should be noted that these estimates reflect costs and charges among those patients who were subsequently admitted following an ED visit. Other than ED costs or charges, no other component costs were reported separately in the included studies. Furthermore, societal costs were not reported in any of the included studies.

\section{CLINICAL BURDEN OF HHF}

The mean (SD) LOS per HHF ranged from 4.3 (3.7) days for adults with acute decompensated HF (mean age, 71.1 years; single-center population-based study) to 7.1 (7.6) days among older adults with HF (mean age, 81 years; Medicare; Table 1). ${ }^{25,33}$ The median 


\begin{tabular}{|c|c|c|c|c|c|c|c|}
\hline Citation & Patient population & Subgroup & $\mathbf{n}$ & $\begin{array}{l}\text { LOS } \\
\text { Mean (SD) } \\
\text { (days) }\end{array}$ & $\begin{array}{l}\text { Case } \\
\text { fatality } \\
(\%)\end{array}$ & $\begin{array}{l}\text { Rehospitalization } \\
\text { definition (\%) }\end{array}$ & $\begin{array}{c}\text { Rehospitalization } \\
\text { estimate (\%) }\end{array}$ \\
\hline $\begin{array}{l}\text { Akintoye, } \\
2017^{13}\end{array}$ & $\begin{array}{l}\text { Adults with primary diagnosis } \\
\text { of } \mathrm{HF}\end{array}$ & Overall & $1,946,895$ & $\begin{array}{l}5.3 \\
(5.8)\end{array}$ & 3 & NA & NR \\
\hline \multirow{3}{*}{$\begin{array}{l}\text { Akintoye, } \\
2017^{34}\end{array}$} & \multirow{3}{*}{ Adults with $\mathrm{HF}$} & $\begin{array}{l}\text { Government } \\
\text { hospitals }\end{array}$ & 226,815 & $\begin{array}{c}5.3 \\
(7.3) \\
\end{array}$ & 3.3 & NA & NR \\
\hline & & $\begin{array}{l}\text { Private hospitals } \\
\text { (nonprofit) }\end{array}$ & $1,430,240$ & $\begin{array}{l}5.4 \\
(5.8)\end{array}$ & 2.9 & NA & NR \\
\hline & & $\begin{array}{l}\text { Private hospitals } \\
\quad \text { (invest) }\end{array}$ & 289,775 & $\begin{array}{l}5.0 \\
(4.6)\end{array}$ & 2.8 & NA & NR \\
\hline $\begin{array}{l}\text { Akintoye, } \\
2017^{12}\end{array}$ & $\begin{array}{l}\text { Adults with primary diagnosis } \\
\text { of } \mathrm{HF}\end{array}$ & Overall & 578,769 & $\begin{array}{c}5.2 \\
(5.7)\end{array}$ & $3.1^{5}$ & NA & NR \\
\hline \multirow{2}{*}{$\begin{array}{l}\text { Asrani, } \\
2018^{15}\end{array}$} & \multirow{2}{*}{$\begin{array}{l}\text { Adults aged }>25 \text { years with a } \\
\text { primary diagnosis of HF }\end{array}$} & \multirow{2}{*}{ Overall } & \multirow{2}{*}{6,492} & \multirow{2}{*}{$\begin{array}{l}6.2 \\
(6.7) \\
\end{array}$} & \multirow{2}{*}{ NR } & HF-specific 30-day & 21.9 \\
\hline & & & & & & HF-specific 90-day & 31.2 \\
\hline $\begin{array}{l}\text { Chang, } \\
2016^{18}\end{array}$ & Adults with $\mathrm{HF}$ & Overall & 97,901 & $\begin{array}{c}3.2-3.7 \\
(3.1-3.7)^{\mathrm{a}}\end{array}$ & NR & NA & NR \\
\hline \multirow{2}{*}{$\begin{array}{l}\text { Cooper, } \\
2015^{27}\end{array}$} & \multirow{2}{*}{$\begin{array}{l}\text { Adults with new onset or } \\
\text { decompensated HF }\end{array}$} & \multirow{2}{*}{ Overall } & \multirow{2}{*}{62,908} & \multirow{2}{*}{$\begin{array}{l}5.7 \\
(4.7)\end{array}$} & \multirow{2}{*}{4.5} & All-cause 30-day & 23.0 \\
\hline & & & & & & All-cause 1 year & 67.5 \\
\hline $\begin{array}{l}\text { Correa, } \\
2018^{20}\end{array}$ & $\begin{array}{l}\text { Adults with primary diagnosis } \\
\text { of HF with and without dialysis- } \\
\text { requiring acute kidney injury }\end{array}$ & Overall & 841,054 & $\begin{array}{c}5.1 \\
(\mathrm{NR})\end{array}$ & NR & NA & NR \\
\hline $\begin{array}{l}\text { Davis, } \\
2017^{21}\end{array}$ & $\begin{array}{l}\text { Adults aged } \geq 40 \text { years with } \\
\text { primary diagnosis of } \mathrm{HF}\end{array}$ & Overall & 547,068 & $\begin{array}{c}4.0 \\
(2.0-7.0)^{a, e}\end{array}$ & NR & All-cause 30-day & 76.6 \\
\hline $\begin{array}{l}\text { Goel, } \\
2019^{23}\end{array}$ & Adults with $\mathrm{HF}$ & Overall & 807,764 & $\begin{array}{l}5.2 \\
(0.1)^{b}\end{array}$ & 2.8 & NA & NR \\
\hline $\begin{array}{l}\text { Imhoff, } \\
2017^{24}\end{array}$ & $\begin{array}{l}\text { Adults with primary diagnosis } \\
\text { of HF }\end{array}$ & HF only patients ${ }^{d}$ & 56,209 & $\begin{array}{c}4.4 \\
(\mathrm{NR})\end{array}$ & 2.1 & NA & NR \\
\hline $\begin{array}{l}\text { Jackson, } \\
2018^{5}\end{array}$ & $\begin{array}{l}\text { Adults with primary diagnosis } \\
\text { of HF }\end{array}$ & Overall & 978,135 & $\begin{array}{c}3.0 \\
(2.0-6.0)^{\mathrm{a}}\end{array}$ & 2.9 & NA & NR \\
\hline \multirow{6}{*}{$\begin{array}{l}\text { Kilgore, } \\
2017^{25}\end{array}$} & \multirow{6}{*}{$\begin{array}{l}\text { Adults aged } \geq 65 \text { years with at } \\
\text { least } 1 \text { hospitalization with } \mathrm{HF} \\
\text { as the primary diagnosis }\end{array}$} & \multirow{6}{*}{ Overall } & \multirow{6}{*}{63,678} & \multirow{6}{*}{$\begin{array}{c}7.1 \\
(7.6)\end{array}$} & \multirow{6}{*}{ NR } & HF-specific 30 days & 8.4 \\
\hline & & & & & & HF-specific 60 days & 13.4 \\
\hline & & & & & & HF-specific 90 days & 16.7 \\
\hline & & & & & & All-cause 30 days & 22.3 \\
\hline & & & & & & All-cause 60 days & 33.3 \\
\hline & & & & & & All-cause 90 days & 40.2 \\
\hline
\end{tabular}

continued on next page

(IQR) LOS per HHF ranged from 3.0 (4.0) days (mean age, 72.1 years; $n=978,135 ;$ NIS) to 5.0 (5.0) days (sample in which $65 \%$ were aged $\geq 70$ years; SPARCS). ${ }^{5,28}$ Among patients with $\mathrm{HF}$ and renal disease, the mean (SD) LOS per HHF ranged from 4.4 (NR) days among patients with CKD and a history of stroke to 13.8 (NR) days among patients with D-AKI. ${ }^{20,24}$ Mean (SD) LOS per HHF ranged from 3.9 (3.2) to 5.3 (SE: 0.2) days for patients with HFpEF and from 4.7 (4.1) to 7.4 (SE: 0.3) days among patients with HFrEF. ${ }^{27,33}$ No studies reported LOS among patients with $\mathrm{HF}$ and diabetes.

Inpatient case fatality estimates among patients hospitalized for HF ranged from $2.8 \%$ to $7.1 \%$ (Table 1). ${ }^{26,34}$ Case fatality among patients with $\mathrm{HF}$ and CKD was $1.9 \%{ }^{24}$ One study reported case fatality rates of $3.5 \%$ among patients with HFpEF and $4.5 \%$ among patients with HFrEF. ${ }^{33}$ No studies reported case fatality among patients with $\mathrm{HF}$ and diabetes. 


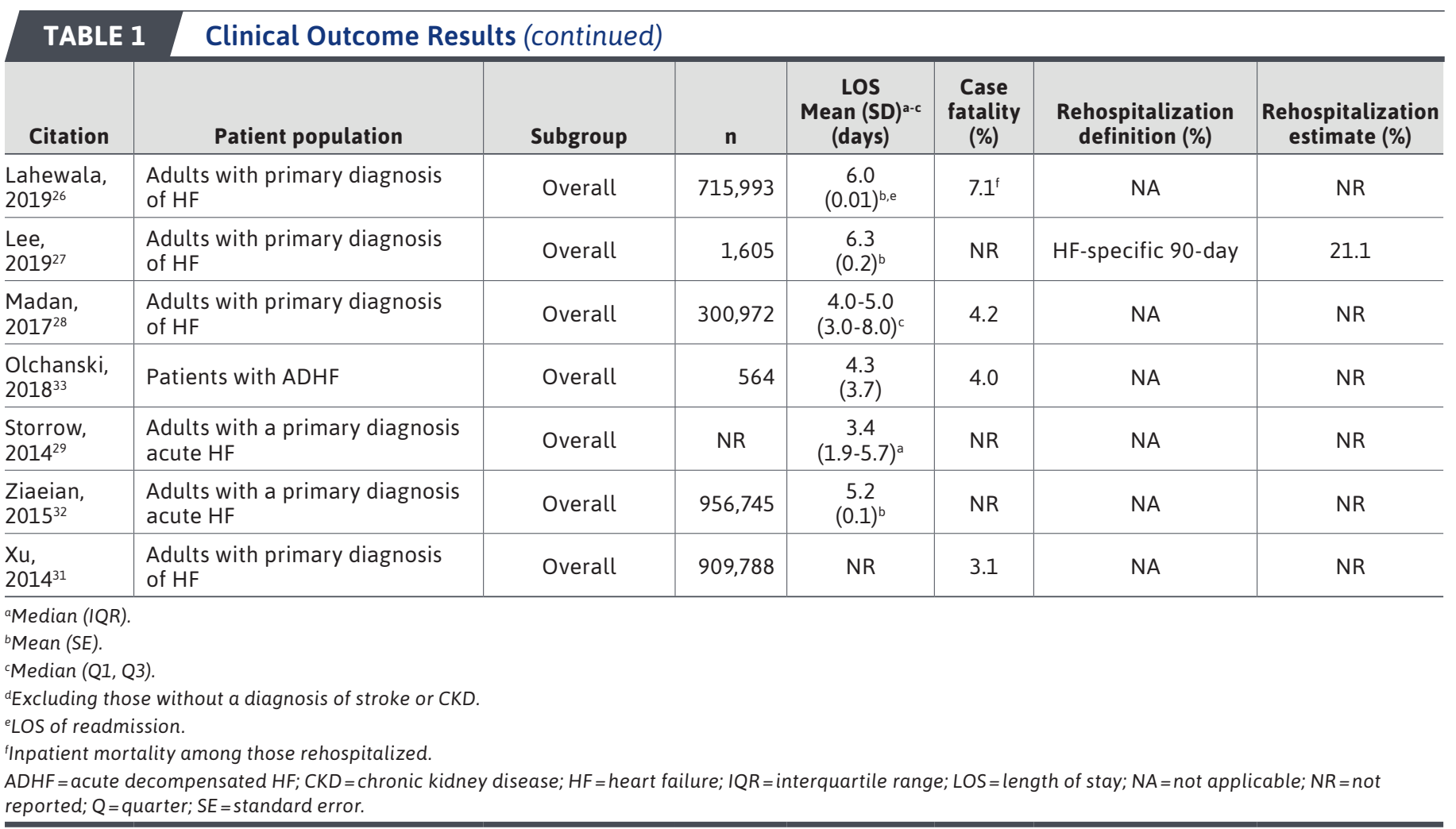

Rates of all-cause 30-day rehospitalization ranged from 21.4\% among patients with a primary HF diagnosis at initial hospitalization (mean age, 74.7 years; 49.3\% male; state inpatient databases [SID]) to $22.3 \%$ among older adults with HF (mean age, 81.1 years; 38.7\% male; Medicare). ${ }^{21,25}$ Rates of HF-specific 30-day rehospitalization were available from 2 studies, which reported that $8.4 \%$ (mean age, 81.1 years; $38.7 \%$ male) and 21.9\% (mean age, $71.1 ; 51.2 \%$ male; Table 1) of patients were rehospitalized within 30 days of the index HF hospitalization. ${ }^{15,25}$ One of the studies (mean age, 81.1 years; Medicare) reporting 30-day rehospitalization also reported rates of rehospitalization at 60 days (all-cause, 33\%; HF-specific hospitalization, 13.4\%) and 90 days (allcause, 40.2\%; HF-specific rehospitalization, 16.7\%). ${ }^{25}$

Two additional studies reported HF-specific 90-day rehospitalization rates of $21.1 \%$ among adults with $\mathrm{HF}$ (mean age, 66.1 years; UHC), and 31.2\% among adults aged 25 years or older with HF $(n=6,492$; Table 1 ; Baylor Scott \& White Health system database). ${ }^{15,27}$ The rate of all-cause 30 -day rehospitalization was $12.3 \%$ among the sample of adults with HFrEF. ${ }^{17}$ HF-specific 90-day rehospitalization was $16.9 \%$ for those with HFpEF and 23.1\% for those with HFrEF.
No studies reported rehospitalization among patients with HF and diabetes.

\section{Discussion}

HF is a substantial and growing health problem, with prevalence in the United States projected to increase by nearly $50 \%$ and direct medical costs to more than double between 2012 and 2030.2,4 Hospitalizations are the largest component of direct medical costs in HF, accounting for up to three-quarters of total costs. ${ }^{6}$ While other recent literature reviews have summarized estimates of HF costs, and 1 recent review included a synthesis of costs for $\mathrm{HHF},{ }^{8}$ to our knowledge this study is the first to present costs per HHF episode along with estimates of the clinical burden of HHF in the United States.

The findings from this SLR demonstrate the substantial economic burden of HHF, with median inpatient costs ranging from $\$ 7,094$ to $\$ 9,769$ and mean costs ranging from $\$ 10,737$ to $\$ 17,830$, per HHF. ${ }^{26,28,33,34}$ From a broader perspective, based on the annual number of hospitalizations for $\mathrm{HF}$ in the United States $(\mathrm{N}=978,135),{ }^{5}$ and the maximum mean cost per hospitalization identified in this review $(\$ 17,830),{ }^{26}$ 


\section{FIGURE 4 Mean Cost per HHF Among Patients With CKD, HFrEF, or HFpEF}

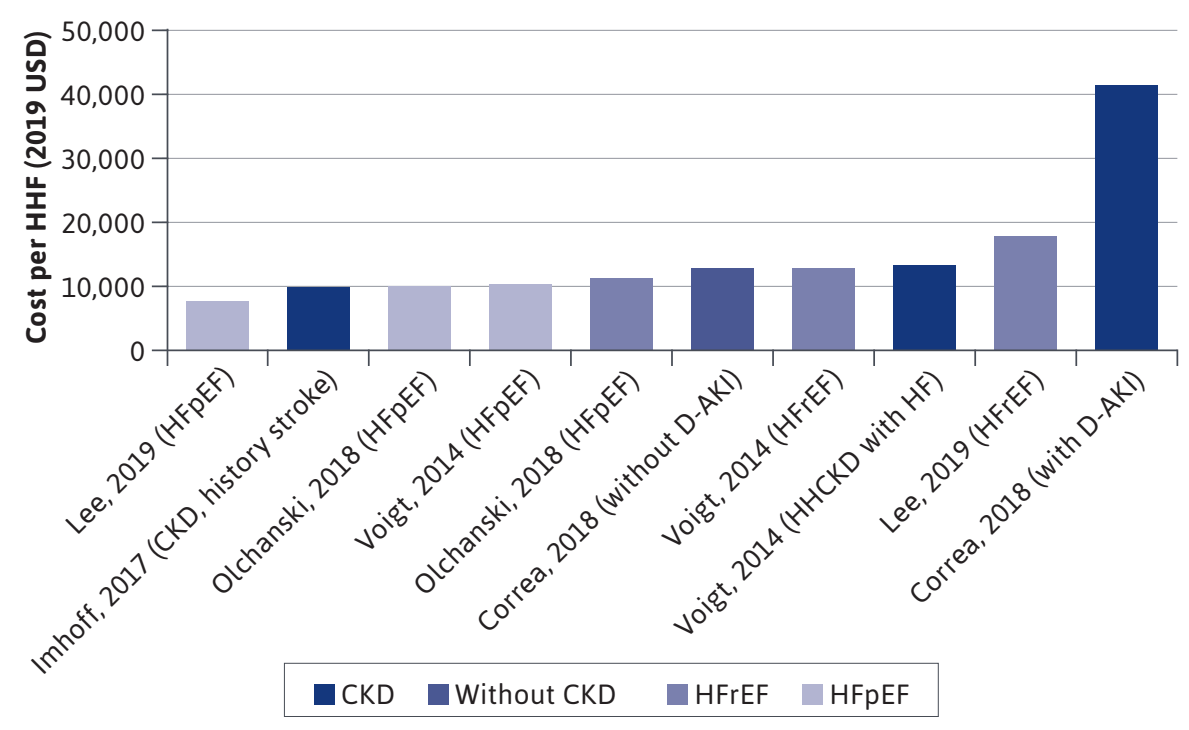

$C K D=$ chronic kidney disease; $D-A K I=$ dialysis-requiring acute kidney injury; HFpEF=heart failure with preserved ejection fraction; $\mathrm{HFrEF}=$ heart failure with reduced ejection fraction; $\mathrm{HHF}=$ hospitalization for heart failure; USD = United States dollars.

HF-related inpatient costs alone could translate to up to $\$ 18$ billion annually in the United States.

Multiple factors may contribute to variability in estimates of the economic burden of HHF. Hospitalization costs and charges for HF would be largely driven by the corresponding LOS estimates, which ranged from a mean of 4 to 7 days (median, 3 to

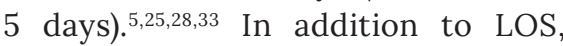
other factors that may impact cost or charge estimates include the severity of $\mathrm{HF}$ and the percentage of patients with comorbidities, which varied between included studies. Further, there was heterogeneity among studies in design, HF case identification criteria, costing methodology, primary payer type, hospital type, and geographic area covered. Studies differed in whether mean or median costs or charges were reported, which limited comparability and synthesis of the estimates. As expected, HHF charges were considerably higher than cost estimates: median charge ranged from approximately $\$ 22,000$ to $\$ 40,000$ and mean charge ranged from approximately $\$ 50,500$ to $\$ 51,000$, per hospitalization. ${ }^{5,23,34}$

The majority of studies included populations with a mixture of primary payer types but did not report costs or charges separately by payer type, which limited the comparability of HHF costs or charges by payer type. For the subgroup of patients with HF and renal disease, 1 study reported mean costs of over $\$ 41,000$ per D-AKI hospitalization among patients with both HF and D-AKI. ${ }^{20}$ While the presence of renal disease has been shown to be associated with increased medical costs among HF patients, ${ }^{35}$ this study population with D-AKI represents patients with more severe renal disease and may not be reflective of costs among those with less severe disease. No studies reported on the cost per hospitalization among patients with HF and diabetes.

From studies that reported clinical outcomes, the percentage of HF patients who died in the hospital ranged from approximately $3 \%$ to $7 \%$ and reached as high as 35\% among the more severe group of patients with HFrEF. ${ }^{17,26,34}$ All-cause 30-day rehospitalization rates ranged from $21 \%$ to $22 \%,{ }^{21,25}$ and the rate of allcause 90-day rehospitalization was $40 \%{ }^{25}$ Rates of HF-specific 30-day rehospitalization ranged from $8 \%$ to $22 \%,{ }^{15,25}$ while rates of $\mathrm{HF}$-specific 90-day rehospitalization ranged from $17 \%$ to $21 \%{ }^{25,27}$

Although more severe patient samples (eg, with HFrEF or comorbid D-AKI) had higher hospitalization costs, there is the potential that hospitalizations with shorter LOS (due to inpatient case fatality) could result in lower hospitalization costs for some severe patients and should be considered when interpreting results. Further, it is also important to note that outcomes occurring after hospitalization, such as rehospitalization, would only be available from patients that survived until the specified time point and did not die in the interim period.

The findings from this review are generally consistent with findings of costs per hospitalization episode from older US studies. For example, a study published in 2014 reported total hospitalization costs for HF and shock ranged from $\$ 5,400$ to $\$ 11,437$ per hospitalization (based on 2012 Medicare data). ${ }^{36}$ A recent global review of overall HF costs published in 2018 reported hospitalization costs ranging from Int $\$ 3,780$ to Int $\$ 25,054.00$ (International dollars, a currency unit used to compare the values of different currencies using a common reference point, the US dollar), ${ }^{37}$ although it 
should be noted that while the 11 studies reported on were largely from the United States, some international studies were also included. In addition, standardizing costs from care provided in different jurisdictions ignores the important differences in patient populations, health care costs, and treatment patterns that underlie the jurisdictionspecific costs.

More recently, a US systematic review of medical costs associated with HF by Urbich et al also included a synthesis of cost and charge estimates for HHF published between January 2014 and March 2020. ${ }^{8}$ The present review extends that work by also considering clinical outcomes, and there are several differences to note between these studies. Urbich et al reported HHF costs ranging from $\$ 7,319$ to $\$ 30,475$ and charges ranging from $\$ 26,393$ to $\$ 51,342$ (2019 USD), not including estimates for subgroups with a particular comorbidity. While the maximum mean HHF cost identified in the present review was $\$ 17,830$ among a sample of adults rehospitalized for HF, the maximum mean HHF cost identified by Urbich et al was $\$ 30,475$ among a sample of HF patients propensity score matched to those with both $\mathrm{HF}$ and protein-energy malnutrition.

Of the 23 studies included in the present review, 12 were included in the Urbich et al review; conversely, Urbich et al included an additional 14 papers reporting HHF costs that were not included here. Reasons for exclusion from the present review included conference abstracts older than 2 years, publication after the search end date, modeling studies, and studies focused on a particular intervention. The present review focused on observational studies of hospitalization for HF, most of which were conducted using large nationally representative databases or databases from health care systems covering large populations.

In addition to the economic burden of HHF, the present study also reported on the clinical burden associated with HHF, including estimates of LOS, rehospitalization, and case fatality. Another more recent review also focused on the economic burden of HF; however, it focused on overall annual costs of HF rather than per hospitalization estimates. ${ }^{6}$ The further exploration of the costs of hospitalizations for $\mathrm{HF}$ and the drivers of these that we conducted will fill a gap in the literature and help provide useful inputs to economic models. Taken together, those reviews and the present synthesis provide valuable insights into the economic and clinical burden of HHF and potential drivers of this burden.

Strengths of this review include the use of rigorous systematic literature review methods to identify hospitalization cost estimates and that the identified estimates were from numerous large well-conducted studies that focused on precisely estimating the economic burden of hospitalization for HF. The majority of data sources were large nationally representative databases or databases from health care systems serving large population centers and study sample sizes were large. In addition, this review focused on studies published within the last 5 years to obtain recent HHF cost estimates that reflect contemporary management practices and treatments for HF.

\section{LIMITATIONS}

As with all evidence syntheses, this review was limited by the validity and reporting accuracy of the included studies. Variability in study designs, data sources, patient populations, and how outcomes were defined across studies contributed to heterogeneity in estimates. These factors were not always well described, which prevented a thorough understanding of their impact on estimates of the economic burden of hospitalizations for HF.

Not all studies included a clear explanation of cost calculation methods, and there was variation in whether mean or median costs or charges were reported, limiting comparability of the estimates. However, the range of observed costs was relatively small despite these differences between studies.

All included studies were database studies, and the limitations inherent to database studies also affected this review. Since claims data were used in many studies, misclassification may have occurred if coding was driven by reimbursement-related factors. In addition, given the sampling frames, findings may not be reflective of outcomes for individuals with or without other types of insurance. However, in the absence of using administrative data, the large sample sizes observed in many of the studies included in this review would not have been possible.

Among the included studies, estimates of direct health care costs were derived from a fairly limited number of data sources (the NIS accounted for approximately $40 \%$ of the total number of data sources); as such, the estimated cost per HHF did not vary particularly widely across studies. Nonetheless, these large datasets remain an excellent choice for understanding US hospital costs and are widely used for pharmacoepidemiologic and pharmacoeconomic research. And, as all studies using these data focused on different time periods, there was no direct duplication of samples.

Finally, because this review was focused on hospitalization costs for HF among large representative samples, studies that did not report cost per hospitalization episode were excluded, as were studies that focused on research questions that negated the usefulness of the sample in reference to the objectives of the present review. The focus on per hospitalization costs in these studies seemed 
to preclude the more detailed exploration of component costs typically found in studies of annual or lifetime costs.

\section{Conclusions}

The findings of this review help characterize the contemporary economic burden of hospitalizations for $\mathrm{HF}$ in the United States, and the clinical findings from the same studies help provide information on the severity of included populations, data on the distribution of other patient characteristics that could be drivers of the economic burden, and context for the economic findings. The economic burden of hospitalizations for $\mathrm{HF}$ is substantial, with mean HF-specific inpatient costs ranging from $\$ 10,737$ to $\$ 17,830$ per hospitalization. When multiplied by the average number of individuals hospitalized for $\mathrm{HF}$ in the United States in a year, this translates to an estimated direct cost of up to \$18 billion for hospitalization costs alone.

Variation exists in the estimates of costs for HHF, and further research into cost drivers of these hospitalizations is warranted to understand potential ways to reduce the associated costs. The results of this synthesis may be useful for economic modeling purposes and to help understand the potential value of new therapies that can decrease the need for hospitalization in individuals with HF.

\section{DISCLOSURES}

This study was funded by Boehringer Ingelheim Pharmaceuticals. Osenenko, Deighton, and Szabo are employees of Broadstreet HEOR, which received funds from Boehringer Ingelheim Pharmaceuticals for this work. Kuti and Pimple are employees of Boehringer Ingelheim Pharmaceuticals.
This study was presented in abstract form at the 2020 American Heart Association (AHA) Quality of Care and Outcomes Research (QCOR) 2020 Scientific Sessions (May 15-16, Virtual Meeting).

\section{REFERENCES}

1. Virani SS, Alonso A, Aparicio HJ, et al. Heart disease and stroke statistics-2021 update: a report from the American Heart Association. Circulation. 2021;143(8):e254-e743.

2. Heidenreich PA, Albert NM, Allen LA, et al. Forecasting the impact of heart failure in the United States: a policy statement from the American Heart Association. Circ Heart Fail. 2013;6(3):606-19.

3. Khera RP, Pandey A, Ayers, C R, et al. Contemporary epidemiology of heart failure in fee-for-service medicare beneficiaries across healthcare settings. Circ Heart Fail. 2017;10(11):e004402.

4. Benjamin EJ, Blaha MJ, Chiuve SE, et al. Heart disease and stroke statistics-2017 update: a report from the American Heart Association. Circulation. 2017;135(10):e146-e603.

5. Jackson SL, Tong X, King RJ, Loustalot F, Hong Y, Ritchey MD. National burden of heart failure events in the United States, 2006 to 2014. Circ Heart Fail. 2018;11(12):e004873.

6. Giles L, Freeman C, Field P, Sörstadius E, Kartman B. Humanistic burden and economic impact of heart failure-a systematic review of the literature [version 2; peer review: 1 approved, 1 approved with reservations]. F1000Research. 2020;8:859. Accessed December 15, 2021. https://doi. org/10.12688/f1000research.19365.2

7. Global Burden of Cardiovascular Diseases Collaboration, Roth GA, Johnson CO, et al. The burden of cardiovascular diseases among US states, 1990-2016. JAMA Cardiol. 2018;3(5):375-89.

8. Urbich M, Globe G, Pantiri K, et al. A systematic review of medical costs associated with heart failure in the USA (2014-2020). Pharmacoeconomics. 2020;38(11):1219-36.
9. Bai G, Anderson GF. Extreme markup: the fifty US hospitals with the highest charge-to-cost ratios. Health Aff (Millwood). 2015;34(6):922-28.

10. von Elm E, Altman DG, Egger M, et al. The Strengthening the Reporting of Observational Studies in Epidemiology (STROBE) statement: guidelines for reporting observational studies. Lancet. 2007;370(9596):1453-57.

11. US Bureau of Labor Statistics. Consumer Price Index (CPI) Databases. 2020. Accessed November 7, 2019. https:// www.bls.gov/cpi/data.htm

12. Akintoye E, Briasoulis A, Egbe A, et al. Seasonal variation in hospitalization outcomes in patients admitted for heart failure in the United States. Clin Cardiol. 2017;40(11):1105-11.

13. Akintoye EB, Briasoulis A. Egbe A, et al. Regional variation in mortality, length of stay, cost, and discharge disposition among patients admitted for heart failure in the United States. Am J Cardiol. 2017;120(5):817-24.

14. Akintoye EB, Briasoulis A, Orhurhu V, et al. Impact of hospital ownership on in-patient mortality and cost of hospitalization in patients admitted for heart failure: insight from the National Inpatient Sample (NIS). J Am Coll Cardiol. 2017;69 (11 Suppl 1):770.

15. Asrani SKK, Kouznetsova M, Ogola G, et al. Increasing health care burden of chronic liver disease compared with other chronic diseases, 2004-2013. Gastroenterology. 2018;155(3):719-29.e714.

16. Basnet S, Dhital R, Tharu B, Poudel DR, Donato A. Comparison of outcomes after hospitalization among heart failure patients with and without history of heart transplantation. Transplant Proc. 2018;50(10):3720-22.

17. Bress APK, King JB, Brixner, D et al. Pharmacotherapy treatment patterns, outcomes, and health resource utilization among patients with heart failure with reduced ejection fraction at a U.S. academic medical center. Pharmacotherapy. 2016;36(2):174-86. 
18. Chang DW, Shapiro MF. Association between intensive care unit utilization during hospitalization and costs, use of invasive procedures, and mortality. JAMA Intern Med. 2016;176(10):1492-99.

19. Cooper LBH, Hammill, BG; Sharma PP, et al. Differences in health care use and outcomes by the timing of in-hospital worsening heart failure. Am Heart J. 2015;170(6):1124-32.

20. Correa A, Patel A, Chauhan K, et al. National trends and outcomes in dialysis-requiring acute kidney injury in heart failure: 2002-2013. J Card Fail. 2018;24(7):442-50.

21. Davis JD, Olsen M A, Bommarito, K, et al. All-payer analysis of heart failure hospitalization 30-day readmission: comorbidities matter. Am J Med. 2017;130(1):93.e99-93.e28.

22. Fitch K, Lau J, Engel T, Medicis JJ, Mohr JF, Weintraub WS. The cost impact to Medicare of shifting treatment of worsening heart failure from inpatient to outpatient management settings. Clinicoecon Outcomes Res. 2018;10:855-63.

23. Goel A, Paydak H, Mehta J. Regional differences in epidemiology and outcomes of heart failure admissions across the United States [abstract]. Circulation. 2019;139(Suppl 1):AP135. Accessed December 15, 2021. https://doi. org/10.1161/circ.139.suppl 1.P135
24. Imhoff RJ, Thomas JT. The inpatient burden of Medicare patients with heart failure, chronic kidney disease and a history of stroke: a retrospective database analysis. Value Health. 2017;20(5):A268. Accessed January 3, 2022. https://www. valueinhealthjournal.com/article/S10983015(17)30251-6/pdf? returnURL=https\% 3A\%2F\%2Flinkinghub.elsevier.com\%2Fret rieve\%2Fpii\%2FS1098301517302516\%3Fsho wall\%3Dtrue

25. Kilgore M, Patel HK, Kielhorn A, Maya JF, Sharma P. Economic burden of hospitalizations of Medicare beneficiaries with heart failure. Risk Manag Healthc Policy. 2017;10:63-70.

26. Lahewala S, Arora S, Tripathi B, et al. Heart failure: same-hospital vs. differenthospital readmission outcomes. Int J Cardiol. 2019;278:186-91.

27. Lee WC, Serag H, Ohsfeldt RL, et al. Racial disparities in type of heart failure and hospitalization. J Immigr Minor Health. 2019;21(1):98-104.

28. Madan SS, Sims D, Saeed O, Patel SR, Shin JJ, Jorde UP. Association of centre volume and in-hospital mortality in heart failure hospitalisations. Postgrad Med J. 2017;93(1103):534-40.

29. Storrow AB, Jenkins CA, Self WH, et al. The burden of acute heart failure on U.S. emergency departments. JACC Heart Fail. 2014;2(3):269-77.

30. Voigt J, Sasha John M, Taylor A, Krucoff M, Reynolds MR, Gibson MC. A reevaluation of the costs of heart failure and its implications for allocation of health resources in the United States. Clin Cardiol. 2014;37(5):312-21.
31. Xu XL, Li SX, Lin H, et al.

"Phenotyping" hospital value of care for patients with heart failure. Health Serv Res. 2014;49(6):2000-16.

32. Ziaeian B, Sharma PP, Yu TC, Johnson K W, Fonarow, GC. Factors associated with variations in hospital expenditures for acute heart failure in the United States. Am Heart J. 2015;169(2): 282-89.e215.

33. Olchanski NV, Vest AR, Cohen JT, Neumann PJ, DeNofrio D. Cost comparison across heart failure patients with reduced and preserved ejection fractions: analyses of inpatient decompensated heart failure admissions. Int J Cardiol. 2018;261:103-08.

34. Akintoye E, Briasoulis A, Egbe A, et al. Effect of hospital ownership on outcomes of heart failure hospitalization. Am J Cardiol. 2017;120(5):831-87.

35. United States Renal Data System. 2020 USRDS annual data report: epidemiology of kidney disease in the United States. National Institutes of Health, National Institute of Diabetes and Digestive and Kidney Diseases. 2020. Accessed August 31, 2021. https://adr.usrds.org/2020.

36. National average costs by department for heart failure and shock (revisited). Healthc Financ Manage. 2014;68(3): 134-35.

37. Shafie AA, Tan YP, Ng CH. Systematic review of economic burden of heart failure. Heart Fail Rev. 2018;23(1):131-45. 